\title{
Über das Verhalten des Kreatins bei der Autolyse.
}

\author{
III. Mitteilung. ${ }^{1}$ )
}

Von

A. Rothmann, Assistent des Instituts.

(Aus dem pharmakologischen Institute der Universität Heidelberg.)

(Der Redaktion zugegangen am 11. August 1908.)

In einer größeren Arbeit über Kreatin und Kreatinin berichtet E. Mellanby ${ }^{2}$ ) auch über das Verhalten der beiden Körper bei der Autolyse. Er kommt dabei zu Resultaten, die in Widerspruch stehen zu den von Gottlieb und Stangassinger erhaltenen Ergebnissen. Aus den früheren Mitteilungen derselben geht hervor, daß Kreatin und Kreatinin bei der Autolyse der Organe zerstört oder weiter umgewandelt werden, daß aber einzelnen Organen auch die Fähigkeit zukommt, Kreatin und Kreatinin zu bilden. Gleichzeitig beobachteten sie die Entstehung von Kreatinin aus zugesetztem Kreatin. Im Gegensatz zu diesen Befunden behauptet Mellanby: ${ }^{3}$ ) *In all autolysis experiments kept rigorously clear of bacterial action and where precautions were taken to prevent the conversion of creatin and creatinin by heating, creatin and creatinin have remained unaffected.»

Aus diesen Befunden Mellanbys ergibt sich vor allem der Einwand, daß die in den Versuchen von Gottlieb und Stangassinger beobachtete Zerstörung resp. Umwandlung zugesetzten Kreatins nicht auf den Vorgängen der Autolyse, sondern auf Bakterienwirkung beruht. Um diesen Einwand zu prüfen, habe ich auf Veranlassung von Herrn Prof. Gottlieb

1) Vgl. R. Gottlieb und R. Stangassinger, Diese Zeitschrift, Bd. LII, S. 1, und R. Stangassinger, Bd. LV, S. 295.

2) Journal of Physiology, Bd. XXXVI, S. 447.

8) loc. cit., S. 465. 
die früher mitgeteilten Autolyseversuche unter sorgfältigstem Ausschluß von Bakterien und unter ständiger Kontrolle von bakteriologischer Seite nochmals wiederholt und dabei auch die methodischen Einwände Mellanbys berücksichtigt.

Ich gehe zunächst auf diejenigen meiner Versuche ein, die sich auf die Veränderung des Gesamtkreatinins (Kreatin plus Kreatinin) bei der Autolyse beziehen. Zur Bestimmung des Gesamtkreatinins haben Gottlieb und Stangassing er das Kreatin durch 3 stündiges Erhitzen in einer Konzentration von 2,2\% Salzsäure auf siedendem Wasserbad in Kreatinin umgewandelt. Dies Verfahren hat sich auch in meinen Versuchen sehr gut bewährt. Es muß aber betont werden, daß die zur quantitativen Umwandlung von Kreatin in Kreatinin nötige Säuremenge je nach dem chemischen Milieu, in dem sich das Kreatin befindet, verschieden stark bemessen werden muß. So macht schon Jaffé ${ }^{1}$ ) darauf aufmerksam, daß das Kreatin im Harne durch die Gegenwart anderer Körper vor dem zerstörenden Einfluß der siedenden Säure geschützt wird, und daß deshalb eine energischere Einwirkung der Salzsäure zur Umwandlung des Kreatins im Harne anwendbar ist und erst zur vollständigen Überführung in Kreatinin führt, als bei reinen Kreatinlösungen. Ähnliche Beobachtungen machten auch Gottlieb und Stangassinger. ${ }^{2}$ ) Im Vergleich zu reinen Kreatinlösungen konnte die Säure bei der Behandlung der Autolyseproben längere Zeit hindurch einwirken, um Kreatin in Kreatinin überzuführen, ohne daß durch Zersetzung Verluste eintraten. Man muß demnach das Optimum der Säurekonzentration, sowie die Art und Dauer der Einwirkung für die verschiedenen Versuchsbedingungen immer erst ermitteln, und die von Gottlieb und Stangassinger gegebene Vorschrift läßt sich nicht ohne weiteres auf die Kreatininbestimmung, z. B. in Fleischextraktlösungen und Harn übertragen. ${ }^{3}$ ) Für die Bestimmung des Kreatinins im Blut

1) Diese Zeitschrift, Bd. XLVIII, S. 436.

2) loc. cit., Bd. LII, S. 13.

${ }^{3}$ ) Vgl. S. Weber, Physiologisches zur Kreatinfrage, Arch. f. exp. Pathol. u. Pharmakol., Bd. LVIII, S. 98, und Dreibholz, Zur Frage cer Kreatinausscheidung im Harn, Inaug.-Diss., Greifswald 1908. 
und in den Filtraten von den Eiweißkoagulis der Autolyseversuche ergibt die 3 stündige Erhitzung in einer Konzentration von 2,2\% iger Salzsäure und das Einengen der sauren Flüssigkeit auf dem Wasserbad bis auf ein ganz geringes Volumen gute Resultate. Ich fand bei der methodischen Nachprüfung dieser Vorschrift stets $95-99 \%$ des vorhandenen Kreatins als Kreatinin wieder. Als Belege führe ich die folgenden Analysen in Rinderblut an. Da das präformierte Kreatinin und Kreatin $1 \mathrm{mg}$ oder höchstens $2 \mathrm{mg}$ pro $100 \mathrm{ccm}$ Blut niemals übersteigt, so sind von dem zugesetzten Kreatin $95-99 \%$ wiedergefunden worden.

\section{Tabelle I.}

Gesamtkreatininbestimmungen im Rinderblut und Hundeleber mit Kreatinzusatz.

\begin{tabular}{|c|c|c|c|c|c|c|}
\hline \multirow{3}{*}{$\mathrm{Nr}$} & \multirow{3}{*}{$\begin{array}{l}\text { Menge des } \\
\text { defibr. Blutes } \\
\text { resp. Leber }\end{array}$} & \multicolumn{2}{|c|}{ Zugesetztes Kreatin } & \multirow{3}{*}{$\begin{array}{l}\text { Gesamt- } \\
\text { kreatinin } \\
\text { in } \mathrm{mg}\end{array}$} & \multirow{2}{*}{\multicolumn{2}{|c|}{$\begin{array}{l}\text { Wieder- } \\
\text { gefundenes } \\
\text { Kreatinin }\end{array}$}} \\
\hline & & \multirow{2}{*}{ in $\mathrm{mg}$} & \multirow{2}{*}{$\begin{array}{l}\text { in mg } \\
\text { Kreatinin } \\
\text { aus- } \\
\text { gedrückt }\end{array}$} & & & \\
\hline & & & & & in $\mathrm{mg}$ & in $\%$ \\
\hline I & $100 \mathrm{ccm}$ & ohne & Zusatz & 1,16 & - & 一 \\
\hline II & > & 25 & 21,564 & 21,83 & 20,67 & 95,8 \\
\hline III & , & 25 & 21,564 & 21,77 & 20,61 & 95,6 \\
\hline IV & , & 12,5 & 10,782 & 11,46 & 10,30 & 96,4 \\
\hline V & , & 25 & 21,43 & 22,13 & 20,97 & 97,6 \\
\hline VI & , & 25 & 21,43 & 22,06 & 20,88 & 97,0 \\
\hline VII & $\begin{array}{c}50 \text { ccm Leberextrakt } \\
=65 \mathrm{~g} \text { Leber }\end{array}$ & ohne & Zusatz & 5,45 & - & 一 \\
\hline VIII & , & 50 & 43,14 & 48,50 & 43,05 & 99,2 \\
\hline IX & $\triangle$ & 50 & 43,14 & 48,10 & 42,65 & 98,4 \\
\hline
\end{tabular}

Für die Zuverlässigkeit der Methode der Gesamtbestimmung sprechen ferner eine große Zahl von Parallelbestimmungen, welche ich mit verschiedenen Portionen des gleichen Blutes vorgenommen habe und die recht befriedigende Übereinstimmung zeigen. In der folgenden Tabelle gebe ich einige Gesamtbestimmungen wieder: 
Tabelle II.

Gesamtkreatininbestimmungen im Hundeblut.

\begin{tabular}{r|c|c}
\hline \hline Nr. & \multicolumn{1}{|c|}{ Material } & $\begin{array}{c}\text { Gefundenes Gesamt- } \\
\text { kreatinin in mg }\end{array}$ \\
\hline I & 100 ccm defibr. Carotisblut vom Hunde & 1,968 \\
II & 100, desselben Blutes & 1,972 \\
III & 100 , defibr. Pfortaderblut & 1,602 \\
IV & 100, desselben Blutes & 1,542 \\
V & 100, defibr. Carotisblut & 2,185 \\
VI & 100, desselben Blutes & 2,20
\end{tabular}

Nachdem ich mich so von der Zuverlässigkeit der Methode überzeugt hatte, wiederholte ich die Versuche über das Verhalten von zugesetztem Kreatin bei der Autolyse von Blut, Leber und Niere. Es wurde dabei mit noch größerer Sorgfalt als bei den früheren Versuchen Bakterienwirkung ausgeschlossen. Herr Prof. 0. Neumann am hiesigen hygienischen Institut hatte die große Freundlichkeit, die von uns jeweils angesetzten Proben durch Abimpfen auf Freisein von Bakterien zu prüfen. Wir sprechen ihm auch an dieser Stelle nochmals unseren Dank aus.

In den früheren Versuchen wurde durch gründlichen Toluolzusatz nach der Bereitung der Organextrakte für möglichste Abwesenheit von Bakterien gesorgt. Doch mußten bei unserem Verfahren einer nicht aseptischen Entnahme der Organe, ihrer Zerkleinerung und Verreibung mit Quarzsand bakterielle Verunreinigungen hineingelangen und man erwartete, daß die Sättigung mit Toluol ausreichen würde, um eine Entwickelung der hineingelangten Keime hintanzuhalten. Vor dem Hineingelangen weiterer Keime schützte ein reichlicher Toluolüberschuß, der als deckende Schicht auf der Autolyseprobe stand. Daß in der Tat Bakterienwirkung auf diese Art hintangehalten wurde, dafür sprach die Abwesenheit von Fäulnisgeruch. Ein solcher trat auch in den früheren Versuchen nur sehr selten ein, und solche Versuche wurden verworfen. Dennoch war es nicht ausge- 
schlossen, daß in den Autolyseproben bei der Verarbeitung der Organe anaerobe Keime unterhalb der Toluolschicht zur Entwickelung gelangten und Veränderungen des Kreatins vortäuschten, die nicht von der Autolyse, sondern von dem Lebensprozeß der Bakterien herrührten. Dieser Einwand scheint aber nur bei den Autolyseversuchen möglich. Die weitgehende Veränderung des Gesamtkreatinins, die Gottlieb und Stangassinger schon bei einer 2-4 stündigen Durchleitung von Leber und Niere und bei sofortiger Verarbeitung des durchgeleiteten Blutes beobachteten, ${ }^{1}$ ) läßt sich bei der Kürze der Versuchszeit wohl kaum durch Bakterienwirkung erklären. Dennoch hielt ich es nicht für überflüssig, die Autolyseversuche mit einer peinlichen Vermeidung von Bakterienwirkung $\mathrm{zu}$ wiederholen. Ich bringe im folgenden ein Beispiel meines Vorgehens in etwas ausführlicher Darstellung.

Hund von $17 \mathrm{~kg}$, am Abend vorher zuletzt gefüttert, in Morphiumnarkose durch Verbluten getötet. Die vorher rasierte Haut wird vor dem Verbluten mit Alkohol gereinigt, die Carotis mit sterilen Instrumenten bloßgelegt; das Blut fließt aus steriler Kanüle und Glasröhre direkt in sterile Glasgefäße ein, die reichlich mit Toluol und mit Glasperlen beschickt waren, und wird durch Schütteln defibriniert und mit Toluol gesättigt. In gleicher Weise werden Leber und Niere mit sterilen Instrumenten entnommen, sogleich in sterile Gefaße mit steriler physiologischer Kochsalzlösung und Toluolschicht versenkt. Die Apparate zur Zerkleinerung der Organe und die Koliertücher sind gleichfalls vorher sterilisiert.

Zur Bereitung der Organextrakte wurden die Organe durch die Fleischhackmaschine getrieben, aus der der Organbrei in Toluolkochsalzlösung aufgefangen wurde. Sodann wurde der Organbrei mit Quarzsand gründlich verrieben und dieser dünnflüssige Brei blieb 1-2 Stunden im Eisschrank; darnach wurde er durch Tücher koliert. Die Menge zugesetzter Flüssigkeit betrug im Verhältnis zum Organgewicht $1: 1$.

Die Resultate des Versuches stelle ich in folgenden Tabellen zusammen. Alle Proben haben sich als bakterienfrei erwiesen.

1) Gottlieb u. Stangassinger, Diese Zeitschrift, Bd. LV, S. 322. 
Tabelle III.

Verhalten von Hundeblut mit Kreatinzusatz bei $37^{\circ}$ (je $100 \mathrm{ccm}$ Blut mit Toluol versetzt).

\begin{tabular}{|c|c|c|c|c|c|c|}
\hline \multirow{3}{*}{ Nr. } & \multicolumn{2}{|c|}{ Kreatinzusatz } & \multirow{3}{*}{$\begin{array}{c}\text { Dauer } \\
\text { des } \\
\text { Versuches }\end{array}$} & \multicolumn{3}{|c|}{ Gefundenes Krettinin } \\
\hline & \multirow[t]{2}{*}{ in $\mathrm{mg}$} & \multirow{2}{*}{$\begin{array}{l}\text { in mg } \\
\text { Kreatinin } \\
\text { aus- } \\
\text { gedrückt }\end{array}$} & & \multirow{2}{*}{$\begin{array}{l}\text { Gesamt- } \\
\text { kreatinin } \\
\text { in } \mathrm{mg}\end{array}$} & \multicolumn{2}{|c|}{$\begin{array}{c}\text { Abnahme } \\
\text { des Gesantwertes }\end{array}$} \\
\hline & & & & & in $\mathrm{mg}$ & in $\%$ \\
\hline I & 125 & 107,85 & $\begin{array}{l}\text { sofort unter- } \\
\text { sucht }\end{array}$ & 102,00 & - & - \\
\hline II & 125 & 107,85 & 68 Stunden & 100,00 & 2,00 & 1,96 \\
\hline III & 125 & 107,85 & 116 & 76,76 & 25,24 & 24,74 \\
\hline IV & 125 & 107,85 & 140 & 77,42 & 24,58 & 24,10 \\
\hline V & 125 & 107,85 & 17 Tage & 76,08 & 26,92 & 26,47 \\
\hline
\end{tabular}

Tabelle IV.

Verhalten von Leberextrakt mit Kreatinzusatz bei $37^{\circ}$ (je $50 \mathrm{ccm}$ Leberextrakt, entsprechend $66 \mathrm{~g} \mathrm{Leber}$ vom Iund).

\begin{tabular}{|c|c|c|c|c|c|c|}
\hline \multirow{3}{*}{$\mathrm{Nr}$. } & \multicolumn{2}{|c|}{ Kreatinzusatz } & \multirow{3}{*}{$\begin{array}{c}\text { Dauer } \\
\text { des } \\
\text { Versuches }\end{array}$} & \multicolumn{3}{|c|}{ Gefundenes Krettinin } \\
\hline & \multirow[t]{2}{*}{ in $\mathrm{mg}$} & \multirow{2}{*}{$\begin{array}{l}\text { in mg } \\
\text { Kreatinin } \\
\text { aus- } \\
\text { gedrückt }\end{array}$} & & \multirow{2}{*}{$\begin{array}{l}\text { Gesamt- } \\
\text { kreatinin } \\
\text { in } \mathrm{mg}\end{array}$} & \multicolumn{2}{|c|}{$\begin{array}{c}\text { Abnihme } \\
\text { des Gesantwertes }\end{array}$} \\
\hline & & & & & in $\mathrm{mg}$ & in $\%$ \\
\hline I & 62,5 & 53,925 & $\begin{array}{c}\begin{array}{c}\text { sofort unter- } \\
\text { sucht }\end{array} \\
\text { suct }\end{array}$ & 57,9 & - & - \\
\hline II & 62,5 & 53,925 & n. 68 Stunden & 47,8 & 10,1 & 17,44 \\
\hline III & 62,5 & 53,925 & 116 & 36,16 & 21,74 & 37,63 \\
\hline IV & 62,5 & 53,925 & 140 & 47,92 & 9,98 & 17,23 \\
\hline
\end{tabular}

Tabelle V.

Verhalten von Nierenextrakt mit Kreatinzusatz bei $3^{\circ}$ (je $40 \mathrm{ccm}$ Extrakt, entsprechend $21 \mathrm{~g}$ Niere vom Hud).

\begin{tabular}{|c|c|c|c|c|c|c|}
\hline \multirow{3}{*}{ Nr. } & \multicolumn{2}{|c|}{ Kreatinzusatz } & \multirow{3}{*}{$\begin{array}{c}\text { Dauer } \\
\text { des } \\
\text { Versuches }\end{array}$} & \multicolumn{3}{|c|}{ Gefundenes Kratinin } \\
\hline & \multirow[t]{2}{*}{ in $\mathrm{mg}$} & \multirow{2}{*}{$\begin{array}{l}\text { in mg } \\
\text { Kreatinin } \\
\text { aus- } \\
\text { gedrückt }\end{array}$} & & \multirow{2}{*}{$\begin{array}{l}\text { Gesamt- } \\
\text { kreatinin } \\
\text { in } \mathrm{mg}\end{array}$} & \multicolumn{2}{|c|}{$\begin{array}{c}\text { Abnuhme } \\
\text { des Gesimtwertes }\end{array}$} \\
\hline & & & & & in $\mathrm{mg}$ & in $\%$ \\
\hline I & 62,5 & 53,925 & $\begin{array}{l}\text { sofort unter- } \\
\text { sucht }\end{array}$ & 56,6 & - & - \\
\hline II & 62,5 & 53,925 & 68 Stunden & 50,0 & 6,6 & 11,6 \\
\hline III & 62,5 & 53,925 & 116 & 43,2 & 13,4 & 23,7 \\
\hline
\end{tabular}


Die bakteriologische Untersuchung der einzelnen Proben hat nun ergeben, daß selbst bei einem so sorgfältigem Vorgehen eine Infektion des Versuchsmaterials bei der Entnahme und Verarbeitung der Organe nicht immer zu vermeiden war. Bei einzelnen Proben - sie bildeten aber nur die Ausnahmen wuchsen einige wenige Kolonien, dieselben waren aber immer nur am ersten Tage nachweisbar und nach 2-3 tägiger oder längerer Einwirkung des Toluols waren die Proben bis auf 2 Ausnahmen immer steril. Diese beiden Ausnahmefälle verrieten sich bei längerem Stehen auch deutlich durch Fäulnisgeruch; sie wurden nicht verarbeitet.

In den folgenden Tabellen stelle ich einige weitere mit bakteriologischer Kontrolle vorgenommene Autolyseproben zusammen. Dieselben zeigen in Bestätigung mit den von Gottlieb und Stangassinger gefundenen Ergebnissen stets eine Abnahme des Gesamtkreatinins, d. h. Zerstörung resp. Umwandlung des Kreatins in andere Substanzen. In einzelnen Fällen zeigt sich bei längerer Dauer der Autolyse wieder ein Wachsen des Gesamtwertes. Von besonderer Bedeutung scheint es zu sein, daß im Pfortaderblut schon nach 2 tägiger Autolyse sich eine beträchtliche Zunahme des Gesamtkreatinins nachweisen läßt. Wir schließen daraus, wie auch Stangassinger, auf das Vorhandensein von Vorstufen im Darmblut.

\section{Tabelle VI.}

Verhalten von Hundeblut mit Kreatinzusatz bei $37^{\circ}$

(je $50 \mathrm{ccm}$ Hundeblut mit Toluol versetzt).

\begin{tabular}{c|c|c|c|c|c|c}
\hline \hline \multirow{2}{*}{ Nr. } & \multicolumn{2}{|c|}{ Kreatinzusatz } & Dauer & \multicolumn{2}{|c}{ Gefundenes Kreatinin } \\
\cline { 2 - 7 } & in mg & $\begin{array}{c}\text { in mg } \\
\text { Kreatinin } \\
\text { aus- } \\
\text { gedrückt }\end{array}$ & $\begin{array}{c}\text { des } \\
\text { Versuches }\end{array}$ & $\begin{array}{c}\text { Gesamt- } \\
\text { kreatinin } \\
\text { in mg }\end{array}$ & $\begin{array}{c}\text { Abnahme } \\
\text { des Gesamtwertes }\end{array}$ \\
\cline { 4 - 7 } I & 50 & 43,14 & $\begin{array}{c}\text { sofort unter- } \\
\text { sucht }\end{array}$ & 40,04 & - & - \\
II & 50 & 43,14 & n. 90Stunden & 28,92 & 11,12 & 27,77 \\
III & 50 & 43,14 &, 160, & 32,78 & 7,26 & 18,13
\end{tabular}


A. Rothmann,

Tabelle VII.

Verhalten von Leberextrakt mit Kreatinzusatz bei $37^{\circ}$ (je $50 \mathrm{ccm}$ Extrakt, entsprechend $60 \mathrm{~g}$ Leber vom Hunc).

\begin{tabular}{|c|c|c|c|c|c|c|}
\hline \multirow{3}{*}{$\mathrm{Nr}$. } & \multicolumn{2}{|c|}{ Kreatinzusatz } & \multirow{3}{*}{$\begin{array}{c}\text { Dauer } \\
\text { des } \\
\text { Versuches }\end{array}$} & \multicolumn{3}{|c|}{ Gefundenes Kredinin } \\
\hline & \multirow[t]{2}{*}{ in $\mathrm{mg}$} & \multirow{2}{*}{$\begin{array}{l}\text { in mg } \\
\text { Kreatinin } \\
\text { aus- } \\
\text { gedrückt }\end{array}$} & & \multirow{2}{*}{$\begin{array}{l}\text { Gesamt- } \\
\text { Kreatinin } \\
\text { in } \mathrm{mg}\end{array}$} & \multicolumn{2}{|c|}{$\begin{array}{cl}\text { Abnaime } \\
\text { des Gesantwertess }\end{array}$} \\
\hline & & & & & in $\mathrm{mg}$ & in $\%$ \\
\hline I & 50 & 43,14 & $\begin{array}{l}\text { sofort unter- } \\
\text { sucht }\end{array}$ & 48,80 & 一 & - \\
\hline II & 50 & 43,14 & 64 Stunden & 37,50 & 11,30 & 23,15 \\
\hline III & 50 & 43,14 & 88 & 31,16 & 17,64 & 36,15 \\
\hline IV & 50 & 43,14 & 160 & 36,04 & 12,76 & 26,14 \\
\hline
\end{tabular}

Tabelle VIII.

Verhalten von Leberextrakt mit Kreatinzusatz bei $37^{\circ}$ (je $50 \mathrm{ccm}$ Extrakt $=33 \mathrm{~g}$ Leber vom Hund).

\begin{tabular}{|c|c|c|c|c|c|c|}
\hline \multirow{3}{*}{$\mathrm{Nr}$. } & \multicolumn{2}{|c|}{ Kreatinzusatz } & \multirow{3}{*}{$\begin{array}{c}\text { Dauer } \\
\text { des } \\
\text { Versuches }\end{array}$} & \multicolumn{3}{|c|}{ Gefundenes Krestinin } \\
\hline & \multirow[t]{2}{*}{ in $\mathrm{mg}$} & \multirow{2}{*}{$\begin{array}{l}\text { in mg } \\
\text { Kreatinin } \\
\text { aus- } \\
\text { gedrückt }\end{array}$} & & \multirow{2}{*}{$\begin{array}{c}\text { Gesamt- } \\
\text { Kreatinin } \\
\text { in } \mathrm{mg}\end{array}$} & \multicolumn{2}{|c|}{$\begin{aligned} & \text { Abnaime } \\
& \text { des } \text { Gesantwertess } \\
&\end{aligned}$} \\
\hline & & & & & in $\mathrm{mg}$ & in $\%$ \\
\hline I & 50 & 43,14 & $\begin{array}{l}\text { sofort unter- } \\
\text { sucht }\end{array}$ & 40,70 & - & - \\
\hline II & 50 & 43,14 & n. 90 Stunden & 19,70 & 20,94 & 51,45 \\
\hline III & 50 & 43,14 & $>184$ & 29,28 & 11,42 & 28,06 \\
\hline
\end{tabular}

Tabelle IX.

Verhalten von Pfortaderblut bei $37^{\circ}$.

\begin{tabular}{c|c|c|c|c|c}
\hline \hline Nr. & Material & $\begin{array}{c}\text { Dauer } \\
\text { des } \\
\text { Versuches }\end{array}$ & $\begin{array}{c}\text { Gefundenes } \\
\text { Gesamt- } \\
\text { kreatinin } \\
\text { in mg }\end{array}$ & \multicolumn{2}{|c}{$\begin{array}{c}\text { Zundmme } \\
\text { des Gesantwerteis }\end{array}$} \\
\cline { 3 - 6 } I & $\begin{array}{c}100 \text { ccm defibr. } \\
\text { Pfortaderblut }\end{array}$ & $\begin{array}{c}\text { sofort unter- } \\
\text { sucht } \\
\text { II }\end{array}$ & 2 Tage & 0,890 & in $\%$ \\
\hline
\end{tabular}


Die von Mellanby behauptete Unveränderlichkeit des Kreatins bei der Autolyse dürfte durch diese bakteriologisch kontrollierten Versuche zurückgewiesen sein. Was nun die Entstehung von Kreatinin aus Kreatin anlangt, die Gottlieb und Stangassinger neben weitgehender Zerstörung des Kreatins beobachtet hatten, so macht Mellanby mit Recht den Einwand, daß auch bei der Verarbeitung der Proben schon Kreatinin entstehen kann. Gottlieb und Stangassinger dampften die Filtrate der Essigsäure-Kochsalzkoagulation unter Zusatz von Baryumcarbonat auf siedendem Wasserbad ein, während Mellanby durch Alkohol koaguliert und das Filtrat bei einer $37^{\circ}$ nicht übersteigenden Temperatur eindampft, nochmals mit 75\% igem Alkohol extrahiert und wieder abdampft. Es muß von vornherein zugegeben werden, daß der allerdings umständlicheren Methode von Mellanby der Vorzug gebührt. Bei einer Substanz, die schon durch Erhitzen ihrer neutralen wässerigen Lösung in ihr Anhydrid übergeführt wird, läuft man immer Gefahr, auch durch eine kurzdauernde Hitzekoagulation mit nachfolgendem Eindampfen bei höherer Temperatur eine Umwandlung herbeizuführen, welche die Veränderung bei der Autolyse vortäuscht. In der Tat habe ich bei methodischen Versuchen gefunden, daß der Kreatininwert bei Anwendung der Hitzekoagulation um 4-5\% zu hoch ausfallen kann. Jedenfalls ist die Kreatininbestimmung nach dem Verfahren von Gottlieb und Stangassinger schwer zu beherrschen. Die von Mellanby angegebene Methode ergab auch mir korrekte Werte.

$$
\text { Tabelle X. }
$$

Kreatininbestimmungen im Rinderblut mit Zusatz von Kreatin und Kreatinin.

\begin{tabular}{r|c|c|c|c|c|c|c|c}
\hline \hline Nr. & $\begin{array}{c}\text { Menge } \\
\text { des defibr. } \\
\text { Rinder- } \\
\text { blutes } \\
\text { in ccm }\end{array}$ & in mg & $\begin{array}{c}\text { Kreatinzusatz } \\
\text { Kreating } \\
\text { aus- } \\
\text { gedrückt }\end{array}$ & $\begin{array}{c}\text { Krea- } \\
\text { tinin- } \\
\text { zusatz } \\
\text { in mg }\end{array}$ & $\begin{array}{c}\text { Gefun- } \\
\text { denes } \\
\text { Krea- } \\
\text { tinin } \\
\text { in mg }\end{array}$ & $\begin{array}{c}\text { Ge- } \\
\text { fundenes } \\
\text { Gesamt- } \\
\text { kreatinin } \\
\text { in mg }\end{array}$ & \multicolumn{2}{|c|}{$\begin{array}{c}\text { Wieder- } \\
\text { gefundenes } \\
\text { Kreatinin }\end{array}$} \\
\hline in mg & in \% \\
\hline I & 100 & - & - & - & - & 1,16 & - & - \\
II & 100 & 50 & 43,14 & - & Spuren & 44,26 & 43,10 & 99,8 \\
III & 100 & 50 & 43,14 & - & 2 & 44,14 & 42,98 & 99,6 \\
IV & 50 & - & - & 10 & 9,95 & - & 9,95 & 99,5 \\
V & 50 & - & - & 10 & 10,03 & - & 10,03 & 100,0
\end{tabular}


Der von Mellanby angegebenen Methode der Alkoholkoagulation gebührt somit für die Kreatininbestimmung der Vorzug, während für die Bestimmung des Gesamtkreatinins das schnellere Verfahren der Hitzekoagulation nach Gottlieb und Stangassinger ausreichend ist. Ich benutzte deshalb Mellanbys Methode, um die Resultate von Gottlieb und Stangassinger über die Entstehung von Kreatinin aus Kreatin bei der Autolyse der Organe mit Kreatinzusatz nochmals nachzuprüfen.

Ich gebe einen solchen Versuch wieder.

\section{Tabelle XI.}

Verhalten von Nierenextrakt mit Kreatinzusatz bei $37^{\circ}$

(mit je $40 \mathrm{ccm}$ entsprechend $30 \mathrm{~g}$ Niere vom Hund).

\begin{tabular}{|c|c|c|c|c|c|c|c|c|c|}
\hline \multirow{3}{*}{ Probe } & \multicolumn{2}{|c|}{ Kreatinzusatz } & \multirow{3}{*}{$\begin{array}{c}\text { Dauer } \\
\text { des } \\
\text { Ver- } \\
\text { suches }\end{array}$} & \multicolumn{6}{|c|}{ Gefundenes Kreatinin } \\
\hline & \multirow[t]{2}{*}{ in $\mathrm{mg}$} & \multirow{2}{*}{$\begin{array}{l}\text { in mg } \\
\text { Krea- } \\
\text { tinin } \\
\text { ausge- } \\
\text { drückt }\end{array}$} & & \multirow{2}{*}{$\begin{array}{l}\text { Krea- } \\
\text { tinin } \\
\text { als sol- } \\
\text { ches }\end{array}$} & \multicolumn{2}{|c|}{$\begin{array}{l}\text { Kreatinin- } \\
\text { vermehrung }\end{array}$} & \multirow{2}{*}{$\begin{array}{l}\text { Ge- } \\
\text { samt- } \\
\text { krea- } \\
\text { tinin } \\
\text { in } \mathrm{mg}\end{array}$} & \multicolumn{2}{|c|}{$\begin{array}{l}\text { Abnahme des } \\
\text { Gesamtwertes }\end{array}$} \\
\hline & & & & & in $\mathrm{mg}$ & in $\%$ & & in $\mathrm{mg}$ & in $\%$ \\
\hline I. & 100 & 86,28 & $\begin{array}{c}\text { sofort } \\
\text { untersucht }\end{array}$ & 2,512 & - & - & 87,56 & - & - \\
\hline II. & 100 & 86,28 & 48 Std. & 7,168 & 4,656 & 5,39 & 71,36 & 16,20 & 18,5 \\
\hline III. & 100 & 86,28 & 48 , & 6,922 & 4,41 & 5,23 & 69,22 & 18,32 & 20,9 \\
\hline
\end{tabular}

Auch bei Anwendung der Methode Mellanbys tritt also Kreatinin in nachweisbarer Menge bei der Autolyse auf. Danach wird man wohl auch die Resultate der früheren Versuche im wesentlichen für richtig halten müssen, nur ist es möglich. daß sowohl die Menge des präformierten Kreatinins als die Werte des bei der Autolyse neu entstandenen Kreatinins ur. einige Prozente $\mathrm{zu}$ hoch erscheinen.

Worauf es beruht, daß die Versuche Mellanbys sowoh: in bezug auf die fermentative Zerstörung des Kreatins bei der Autolyse, wie auch in bezug auf Kreatininbildung negativ ausgefallen sind, entzieht sich unserer Beurteilung. Ich hielt es jedenfalls nicht für überflüssig, einige Versuche möglichst unter Einhaltung der von Mellanby mitgeteilten Bedingungen anzustellen. 
Über das Verhalten des Keratins bei der Autolyse. III. 141

$$
\text { Tabelle XII. }
$$

Verhalten von Leberextrakt mit Kreatinzusatz bei $37^{\circ}$

(mit je $20 \mathrm{ccm}$ entsprechend $12 \mathrm{~g}$ Leber einer Katze).

\begin{tabular}{|c|c|c|c|c|c|c|c|c|c|}
\hline \multirow{3}{*}{ Probe } & \multicolumn{2}{|c|}{ Kreatinzusatz } & \multirow{3}{*}{$\begin{array}{l}\text { Dauer } \\
\text { des } \\
\text { Ver- } \\
\text { suches }\end{array}$} & \multicolumn{6}{|c|}{ Gefundenes Kreatinin } \\
\hline & \multirow[t]{2}{*}{ in $\mathrm{mg}$} & \multirow{2}{*}{$\begin{array}{l}\text { in mg } \\
\text { Krea- } \\
\text { tinin } \\
\text { ausge- } \\
\text { drückt }\end{array}$} & & \multirow{2}{*}{$\begin{array}{c}\text { Krea- } \\
\text { tinin } \\
\text { als sol- } \\
\text { ches }\end{array}$} & \multicolumn{2}{|c|}{$\begin{array}{l}\text { Kreatinin- } \\
\text { vermehrung }\end{array}$} & \multirow{2}{*}{$\begin{array}{c}\text { Ge- } \\
\text { samt- } \\
\text { krea- } \\
\text { tinin } \\
\text { in } \mathrm{mg}\end{array}$} & \multicolumn{2}{|c|}{$\begin{array}{l}\text { Abnahme des } \\
\text { Gesamtwertes }\end{array}$} \\
\hline & & & & & in $\mathrm{mg}$ & in $\%$ & & in $\mathrm{mg}$ & in $\%$ \\
\hline I. & $\begin{array}{r}\text { nichts } \\
\text { fü }\end{array}$ & zuge- & $\begin{array}{c}\text { sofort } \\
\text { untersucht }\end{array}$ & Spuren & 一 & - & Spuren & - & - \\
\hline II. & 50 & 43,14 & 48 Std. & 1,356 & 1,356 & 3,14 & 24,92 & 18,22 & 42,23 \\
\hline III. & 50 & 43,14 & 48 , & 1,472 & 1,472 & 3,41 & 23,82 & 19,31 & 44,76 \\
\hline
\end{tabular}

Tabelle XIII.

Verhalten von Leberextrakt mit Kreatinzusatz bei $37^{\circ}$ (je $30 \mathrm{ccm}$ Extrakt entsprechend $16 \mathrm{~g}$ Leber einer Katze).

\begin{tabular}{|c|c|c|c|c|c|c|c|c|}
\hline \multirow{3}{*}{ Nr. } & \multicolumn{2}{|c|}{ Kreatinzusatz } & \multirow{3}{*}{$\begin{array}{c}\text { Physio- } \\
\text { logische } \\
\text { Kochsalz- } \\
\text { lösung } \\
\text { in ccm }\end{array}$} & \multirow{3}{*}{$\begin{array}{c}\text { Leber- } \\
\text { ex- } \\
\text { trakt } \\
\text { in } \mathrm{ccm}\end{array}$} & \multirow{3}{*}{$\begin{array}{c}\text { Dauer } \\
\text { des } \\
\text { Ver- } \\
\text { suches }\end{array}$} & \multirow{3}{*}{$\begin{array}{c}\text { Gesamt- } \\
\text { kreatinin- } \\
\text { bestim- } \\
\text { mung }\end{array}$} & \multirow{2}{*}{\multicolumn{2}{|c|}{$\begin{array}{l}\text { Abnahme } \\
\text { des Gesamt- } \\
\text { wertes }\end{array}$}} \\
\hline & \multirow{2}{*}{ in $\mathrm{mg}$} & \multirow{2}{*}{$\begin{array}{l}\text { in mg } \\
\text { Kreatinin } \\
\text { ausge- } \\
\text { drückt }\end{array}$} & & & & & & \\
\hline & & & & & & & in $\mathrm{mg}$ & in $\%$ \\
\hline I. & 50 & 43,15 & 55 & - & 7 & 41,54 & - & - \\
\hline II. & 50 & 43,15 & 25 & 30 & & 35,21 & 6,33 & 15,1 \\
\hline III. & 50 & 43,15 & 25 & 30 & & 33,75 & 7,79 & 18,3 \\
\hline
\end{tabular}

Tabelle XIV.

Verhalten von Leberextrakt mit Kreatinzusatz bei $37^{\circ}$ (je $30 \mathrm{ccm}$ Extrakt entsprechend $17 \mathrm{~g}$ Leber einer Katze).

\begin{tabular}{|c|c|c|c|c|c|c|c|c|}
\hline \multirow{3}{*}{ Nr. } & \multicolumn{2}{|c|}{ Kreatinzusatz } & \multirow{3}{*}{$\begin{array}{c}\text { Physio- } \\
\text { logische } \\
\text { Kochsalz- } \\
\text { lösung } \\
\text { in ccm }\end{array}$} & \multirow{3}{*}{$\begin{array}{c}\text { Leber- } \\
\text { ex- } \\
\text { trakt } \\
\text { in ccm }\end{array}$} & \multirow{3}{*}{\begin{tabular}{|c|} 
Dauer \\
des \\
Ver- \\
suches
\end{tabular}} & \multirow{3}{*}{$\begin{array}{c}\text { Gesamt- } \\
\text { kreatinin } \\
\text { bestim- } \\
\text { mung }\end{array}$} & \multirow{2}{*}{\multicolumn{2}{|c|}{$\begin{array}{l}\text { Abnahme } \\
\text { des Gesamt- } \\
\text { wertes }\end{array}$}} \\
\hline & \multirow{2}{*}{ in $\mathrm{mg}$} & \multirow{2}{*}{$\begin{array}{c}\text { in mg } \\
\text { Kreatinin } \\
\text { ausge- } \\
\text { drückt }\end{array}$} & & & & & & \\
\hline & & & & & & & in $\mathrm{mg}$ & in $\%$ \\
\hline I. & 50 & 43,15 & 55 & - & 7 & 42,54 & - & - \\
\hline II. & 50 & 43,15 & 25 & 30 & & 35,20 & 7,34 & 17,2 \\
\hline III. & 50 & 43,15 & 25 & 30 & Tage & 35,84 & 6,70 & 15,7 \\
\hline
\end{tabular}


142 Rothmann, Verhalten des Keratins bei der Autolyse. III

Während ich mit diesen Untersuchungen beschäftig war, erhielten wir Kenntnis von der Dissertation von Van Horgenhuyze, Utrecht 1908, in der die Autolyseversuche von Gottlieb und Stangassinger gleichfalls einer Nachprüfung anterzogen worden sind. Auch dieser Autor konnte bei der Atolyse von Organen Zerstörung von Kreatin und Zunahme des Kreitinins nachweisen. 